\title{
Letter to Editor
}

\section{Dengue viral infection: An Emerging Infectious Disease in Nepal}

\author{
Sherchand Jatan Bahadur \\ Department of Medical Microbiology, Kathmandu University School of Medical Sciences, \\ Dhulikhel, Kavre, Nepal.
}

Date of Submission: Feb 1, 2020

Received in Revised Form: March 21, 2020

Date of Acceptance: May 03, 2020

Date of Publishing: July 30, 2020

Dengue virus is primarily transmitted by Aedes mosquitos, particularly A. aegypti. Other Aedes species that transmit the disease include A. albopictus, A. polynesiensis and A. scutellaris. ${ }^{1}$

There are five strains of the virus, called serotypes, of which the first four are referred to as DENV-1, DENV-2, DENV-3 and DENV4. The fifth type was announced in 2013. ${ }^{1,2}$

There is an estimate of 390 million dengue virus infections per year globally and 3.9 billion people are at risk of infection with dengue viruses and $70 \%$ of the actual burden is shouldered by Asia. ${ }^{3}$

Although dengue viral infection was detected for first time in Nepal by Sherchand et al., $2001,{ }^{4}$ Dengue hemorrhagic fever case was identified in 2004 in Chitwan in one Japanese tourist who had traveled from Vietnam. ${ }^{5}$ Subsequently larger outbreak occurred in 9 districts of Terai region in 2006 with 23 confirmed dengue cases following the Indian epidemic of DF/DHF in September-October $2006.5,6,7$

Total number of confirmed cases of dengue in Nepal in the year 2017/18 was 2,111 reported from 28 districts. The majority of cases have been reported from Rupandehi (32\%), Jhapa (25\%), Mahottari (20\%) and Sarlahi (6\%). As well there were three confirmed deaths due to dengue, one each from Chitwan, Jhapa and Arghakhanchi. ${ }^{8}$

Dengue symptoms typically begin three to fourteen days after infection. ${ }^{9}$ This may include a high fever, headache, vomiting, muscle and joint pains, and a characteristic skin rash. ${ }^{9,10}$ Recovery generally takes two to seven days. ${ }^{9}$ In a small proportion of cases, the disease develops into severe dengue, also known as dengue hemorrhagic fever, resulting in bleeding, low levels of blood platelets and blood plasma leakage, or into dengue shock syndrome, where dangerously low blood pressure occurs. ${ }^{9,10}$ Infection with one type of virus usually gives lifelong immunity 
to that type, but only short-term immunity to the other three. ${ }^{9}$ Subsequent infections with a different type increase the risk of severe complications. ${ }^{9}$ The World Health Organization's 2009 classification divides dengue fever into two groups: uncomplicated and severe. ${ }^{1,11}$

Humans are the primary host of the virus, ${ }^{1,12}$ but it also circulates in nonhuman primates. ${ }^{13}$ An infection can be acquired via a single bite. ${ }^{14} \mathrm{~A}$ female mosquito that takes a blood meal from a person infected with dengue fever, during the initial 2 to 10 day febrile period, becomes itself infected with the virus in the cells lining its gut. ${ }^{15}$ About 8-10 days later, the virus spreads to other tissues including the mosquito's salivary glands and is subsequently released into its saliva. The virus seems to have no detrimental effect on the mosquito, which remains infected for life. ${ }^{16}$ Aedes aegypti is particularly involved, as it prefers to lay its eggs in artificial water containers, to live in close proximity to humans, and to feed on people rather than other vertebrates. ${ }^{16}$

Dengue can also be transmitted via infected blood products and through organ donation. ${ }^{17,18}$ The risk of severe disease from secondary infection increases if someone previously exposed to serotype DENV-1 contracts serotype DENV-2 or DENV-3, or if someone previously exposed to DENV-3 acquires DENV-2. ${ }^{19}$ Dengue can be life-threatening in people with chronic diseases such as diabetes and asthma. ${ }^{19}$ After mosquito bite the virus enters the skin together with the mosquito's saliva. It binds to and enters white blood cells, and reproduces inside the cells while they move throughout the body. The white blood cells respond by producing a number of signaling proteins, such as cytokines and interferons, which are responsible for many of the symptoms, such as fever, the flu like symptoms, and the severe pains. In severe infection, the virus production inside the body is greatly increased, and many more organs (such as liver and bone marrow) can be affected. Fluid from the bloodstream leaks through the wall of small blood vessels into body cavities due to capillary permeability. As a result, less blood circulates in the blood vessels, and the blood pressure becomes so low that it cannot supply sufficient blood to vital organs. Furthermore, dysfunction of the bone marrow due to infection of the stromal cells leads to reduced numbers of platelets, which are necessary for effective blood clotting; this increases the risk of bleeding, the other major complication of dengue fever. ${ }^{20}$

The diagnosis of dengue is typically made clinically, on the basis of reported symptoms and physical examination; this applies especially in endemic areas. ${ }^{21}$ However, early disease can be difficult to differentiate from other viral infections. ${ }^{22}$ The diagnosis of dengue fever may be confirmed by microbiological diagnosis by virus isolation in cell cultures, nucleic acid detection by PCR, viral antigen detection (such as for NS1) or specific antibodies. ${ }^{1,19}$

Nepal's Dengue Control Programme's goal is to reduce the morbidity and mortality due to dengue fever, dengue hemorrhagic fever (DHF) and dengue shock syndrome (DSS). The objectives are to develop an integrated vector management (IVM) approach for prevention and control and to develop capacity on diagnosis and case management of dengue fever, DHF and DSS and to intensify health education and IEC activities and to strengthen the surveillance system for prediction, early detection, preparedness and early response to 
dengue outbreaks. ${ }^{8}$

Strategies such as early case detection, diagnosis, management and reporting of dengue fever, DHF and DSS, regular monitoring of dengue fever, DHF and DSS cases and surveillance, mosquito vector surveillance in municipalities and integrated vector control approach where a combination of several approaches are directed towards containment and source reduction. ${ }^{8}$ Major activities in 2017/2018 was involvement of trained physicians, nurses, paramedics and laboratory technicians on dengue case detection, diagnosis, management and reporting, orientation of municipality stakeholders in 28 program districts, supplement of rapid diagnostic test kits (IgM), dengue case monitoring and vector surveillance, search and destruction of dengue vector larvae (A. aegypti) in 28 program districts and development and dissemination health education messages. ${ }^{8}$

As of 2019 one version of dengue vaccine is commercially available, known as CYT-TDV, and sold under the brand name Dengvaxia. ${ }^{23}$ The vaccine is only recommended in those who have previously had dengue fever or populations in which most people have been previously infected. ${ }^{23}$ The value of the vaccine is limited by the fact that it may worsen outcomes in those who have not previously been infected. International Anti-Dengue Day is observed every year on 15 June. There are no specific antiviral drugs for dengue; however, maintaining proper fluid balance is important. Treatment depends on the symptoms. Most people with dengue recover without any ongoing problems. ${ }^{1}$ The risk of death among those with severe dengue is $0.8 \%$ to $2.5 \%, 9$ and with adequate treatment this is less than $1 \%{ }^{1}$

\section{REFERENCES}

1. WHO (2009). Dengue Guidelines for Diagnosis, Treatment, Prevention and Control (PDF). Geneva: World Health Organization. ISBN 978-92-4-154787-1.

2. Normile D (October 2013)."Tropical medicine. Surprising new dengue virus throws a spanner in disease control efforts". Science. 342 (6157): 415. doi:10.1126/ science. 342.6157.415. PMID 24159024.

3. Bhatt, S., et al., The global distribution and burden of dengue. Nature, 2013. 496(7446): 504-507

4. Sherchand JB, Pandey BD, Haruki K, Jimba M. Sero-diagnosis of Japanese encephalitis and dengue virus infection from clinically suspected patients of Nepal. J Inst Med. 2001; 18: 18-22.

5. WHO. Dengue: Guidelines for diagnosis, treatment, prevention and control. New Ed. Geneva, World Health organization. 2009

6. Pandey BD, Rai SK, Morita K, Kurane I. First case of dengue virus infection in Nepal. Nep Med Coll J. 2004; 6:157-9.

7. Epidemiology and Disease Control Division (EDCD). Annual report 2006/2007. Department of Health Services (DoHS), Ministry of Health, Government of Nepal. 2008.

8. Epidemiology and Disease Control Division (EDCD). Annual report 2017/2018. Department of Health Services (DoHS), Ministry of Health, Government of Nepal. 2019.

9. "Dengue and severe dengue Fact sheet N¹17”. WHO. May 2015. Archived 
from the original on 2 September 2016. Retrieved 3 February 2016.

10. Kularatne SA, (September 2015). "Dengue fever". BMJ. 351: h4661. doi:10.1136/ bmj.h4661. PMID 26374064.

11. Whitehorn J, Farrar J (2010). "Dengue". British Medical Bulletin. 95: 16173. doi:10.1093/bmb/ldq019. PMID 20616106

12. Gould EA, Solomon T (February 2008). "Pathogenic flaviviruses". Lancet. 371 (9611): 500-9. doi:10.1016/S01406736(08)60238-X. PMID 18262042

13. "Vector-borne viral infections". World Health Organization. Archived from the original on 3 February 2011. Retrieved 17 January 2011.

14. Center for Disease Control and Prevention. "Chapter 5 - dengue fever (DF) and dengue hemorrhagic fever (DHF)". 2010 Yellow Book. Archived from the original on 29 December 2010. Retrieved 23 December 2010.

15. St. Georgiev, Vassil (2009). National Institute of Allergy and Infectious Diseases, NIH (1 ed.). Totowa, N.J.: Humana. p. 268. ISBN 978-1-60327297-1. Archived original from 1 May 2016.

16. Gubler DJ (2010). "Dengue viruses". In Mahy BWJ; Van Regenmortel MHV (eds.). Desk Encyclopedia of Human and Medical Virology. Boston: Academic Press. pp. 372-82. ISBN 978-0-12375147-8. Archived from the original on 9 June 2016.

17. Wilder-Smith A, Chen LH, Massad E, Wilson ME (January 2009). "Threat of dengue to blood safety in dengueendemic countries". Emerging Infectious Diseases. 15 (1): 8-11. doi:10.3201/ eid1501.071097. PMC 2660677. PMID
19116042.

18. Stramer SL, Hollinger FB, Katz LM, Kleinman S, Metzel PS, Gregory KR, Dodd RY (August 2009). "Emerging infectious disease agents and their potential threat to transfusion safety". Transfusion. 49 Suppl 2: 1S-29S. doi:10.1111/j.1537-2995. 2009. 02279.x. PMID 19686562

19. Guzman MG, Halstead SB, Artsob H, Buchy P, Farrar J, Gubler DJ, Hunsperger E, Kroeger A, Margolis HS, Martínez E, Nathan MB, Pelegrino JL, Simmons C, Yoksan S, Peeling RW (December 2010). "Dengue: a continuing global threat". Nature Reviews. Microbiology. 8 (12 Suppl): S7-16. doi:10.1038/ nrmicro2460. PMC 4333201. PMID 21079655

20. Martina BE, Koraka P, Osterhaus AD (October 2009). "Dengue virus pathogenesis: an integrated view". Clinical Microbiology Reviews. 22 (4): 564-81. doi:10.1128/ CMR.00035-09. PMC 2772360. PMID 19822889

21. Whitehorn J, Farrar J (2010). "Dengue". British Medical Bulletin. 95: 16173. doi:10.1093/bmb/ldq019. PMID 20616106

22. Ranjit S, Kissoon N (January 2011). "Dengue hemorrhagic fever and shock syndromes". Pediatric Critical Care Medicine. 12 (1): 90-100. doi:10.1097/ PCC.0b013e3181e911a7. PMID 20639791

23. "Dengue vaccine: WHO position paper, September 2018- Recommendations". Vaccine. 37 (35): 4848-4849. 14 August 2019. doi:10.1016/j.vaccine. 2018.09.063. PMID 30424888 